\title{
MR imaging of intracranial solitary fibrous tumor: a retrospective study of 7 cases
}

\author{
Yongshan Liu, Qian Wang, Taijuan Zhang, Linlin Yang, WenJuan Liang
}

Department of Radiology, Qingdao Haici Medical Group,4 Renmin Road, Shibei District, Qingdao,Shandong Province 266033 China.

\section{Author details:}

Yongshan Liu: Phone: +86 0532-83777631, Fax : +86 0532-83777888, Email: liuys7613@126.com

WenJuan Liang, Phone: +86 0532-83777631, Fax :+86 0532-83777888, Email: lwj5203@yeah.net

\begin{abstract}
Objective: To investigate the MR imaging diagnostic features of intracranial solitary fibrous tumors (ISFTs).

Materials and methods: Seven patients (mean age of 52.9 years; M:F=3:4) with histopathologically proven ISFTs were identified at our institute. Clinical presentations and pathological features were reviewed. MR Imaging findings including signal intensity, gadopentetate dimeglumine enhanced pattern, and diffusion-weighted imaging (DWI) characterization of the tumors were retrospectively evaluated.

Results: Six tumors showed a multi-lobular contour. Five tumors showed heterogeneous signal intensity, and two tumors showed homogeneous signal intensity on T1WI. Low signal intensity linear, curved or interlacing lines were observed within the tumors in all seven cases. Seven tumors demonstrated moderate or strong enhancement, six showed heterogeneous enhancement, and one homogenous enhancement. All tumors showed heterogeneous signal intensity on DWI.A ring-like high signal intensity band distributed around within the tumor was noted in six cases on DWI.

Conclusion: Diagnostic evidence for ISFT on MR image includes heterogeneous signal intensity, intense enhancement of T2 signal intensity, low signal intensity lines within the tumor, heterogeneous signal intensity on DWI and a ring-like band around the tumor on DWI.
\end{abstract}

Keywords: Intracranial Solitary Fibrous Tumor, Magnetic resonance imaging, Diffusion-weighted Imaging.

DOI: https://dx.doi.org/10.4314/ahs.v18i3.39

Cite as: Liu Y, Wang Q, Zhang T, Yang L,Liang W. MR imaging of intracranial solitary fibrous tumor: a retrospective study of 7 cases. Afri Health Sci. 2018;18(3): 799-806. bttps:// dx.doi.org/10.4314/abs.v18i3.39

\section{Introduction}

Intracranial solitary fibrous tumor (ISFT) is a rare neoplasm described in 1996 ${ }^{1}$. ISFTs are typically dural-based, CD34-positive neoplasms of mesenchymal origin, resembling other spindle cell tumors such as fibrous me-
Corresponding author:
WenJuan Liang,
Department of Radiology
Qingdao Haici Medical Group
4 Renmin Road, Shibei District, Qingdao
Shandong Province, China 266033
Phone: +86 0532-83777631
Fax : +86 0532-83777888
Email: lwj5203@yeah.net

ningiomas and hemangiopericytomas. Pathological and immunohistochemical studies usually help to achieve an accurate diagnosis ${ }^{2,3}$.

ISFT is a slow growing tumor that is generally asymptomatic or manifested with similar clinical symptoms to other brain tumors ${ }^{4}$. Because of its rarity, literature information is limited to case reports or small series ${ }^{5-7}$. The paucity of specific characteristics in clinical presentation may responsible for missed diagnoses prior to surgery4. Previous studies showed that CT and MR image were reliable and practical techniques for detection and evaluation of ISFTs. Findings of imaging have been proved to provide important diagnostic clues of ISFTs ${ }^{6,8,9}$. However, the information about imaging feature of ISFTs is limited in the literature. In this study, we retrospectively reviewed seven pathologically proven ISFTs'cases with the purpose to be particularly focused on their specific MR performance.

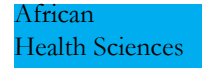

(C) 2018 Liu et al. Licensee African Health Sciences. This is an Open Access article distributed under the terms of the Creative commons Attribution License (https://creativecommons.org/licenses/BY/4.0), which permits unrestricted use, distribution, and reproduction in any medium, provided the original work is properly cited. 


\section{Materials and methods}

Seven patients with pathologically and immunohistochemically proven ISFTs were identified via systematic search of our radiology and pathology department database. Cases were identified from November, 2008 toMarch, 2013. The clinical, pathological and imaging data were retrospectively reviewed. The study included three men and four 4 women with a mean age of 52.1 years (range: 43-64 years). In general, the clinical information was non-specific. The most common presenting symptom of the patients was headache $(100 \%, 7 / 7)$ ranging from 1 month to 3 years. One patient had left-sided weakness, two patients had vertigo with blurring vision, and one patient had headache with hearing disturbances for 1 year.

All seven patients underwent MR imaging examinations. MRI studies were performed on a 1.5 T MR unit (Signa, GE Medical Sytems, WI, US). Imaging protocols included FSE T1 weighted images (T1WI), FSE T2WI, and axial fluid attenuated inversion recovery (FLAIR) sequence. Axial T1-weighted and T2-weighted images were obtained with the following sequences: TR/TE: 1696-1708 ms /18-28ms for T1WI; TR/TE: 3060-480ms-98-110 ms for T2WI; FOV, 18-20 cm; section thickness, $5 \mathrm{~mm}$ with a 1.5 -mm gap; and matrix size, $256 \times 256$. Contrast-enhanced T1-weighted spin-echo images were performed in five patients with (TR/TE: 2642-2643 ms/ 15-17ms) obtained in the axial, sagittal, and coronal planes after intravenous injection of $0.2 \mathrm{mmol} / \mathrm{kg}$ of gadopentetate dimeglumine with a injection rate of $3 \mathrm{ml} / \mathrm{s}$. Diffusion weighted MR imaging (DWI) was performed in all patients with single-shot gradient-echo echo-planar pulse sequence: TR/TE: $5800 \mathrm{~ms} / 8 \mathrm{~ms}$; and b-values of 1000 .
MR images were reviewed by two neuroradiologists (with experience of 8 and 10 years, respectively) at a digital picture-archiving and communication system (PACS) diagnostic workstation. Two readers together reviewed all MR imaging sets, and findings were determined by consensus. MR imaging morphological characteristics included the followinging: location, size, shape, internal component, margin of the lesion, and the change of the adjacent structures were evaluated. The signal intensity, the pattern of enhancement, and the data of DWI were also reviewed. The size of the lesion was measured at its greatest diameter. On the T1- and T2-weighted MR images, the signal intensity abnormalities were categorized as homogeneous or heterogeneous (low, iso, or high) signal intensity. The pattern of intratumoral enhancement was categorized as homogeneous or heterogeneous, and the degree of enhancement was categorized as weak, moderate or strong.

Histopathological and immunohistochemical studies were performed on the excised specimens. Diagnosis of ISFT was established on the basis of histopathologic appearance and immunohistochemical pattern of a diffuse and strong positive reaction for CD34.

\section{Results}

Tumor characteristics of the seven cases are displayed in Table 1. All tumors were extra-axial brain masses, originated from the meninges. Six tumors were supratentorial; one tumor was infratentorial. One tumor was located at the cerebellopontine angle, one tumor at the right temporal- occipital area, one tumor at the left occipital lobe area, two tumors at the frontal lobe areas, and two 2 tumors near the cerebral falx on the right side. The mean maximum diameter of the tumors was $48.1 \mathrm{~mm}$ (range, 13.6-68.8mm). All tumors were well-circumscribed solid, three tumors showed cystic change. Six tumors were multilobular, one small tumor was a smooth unilobular. 
Table 1. The tumor characteristics of the seven intracranial solitary fibrous tumor cases.

\begin{tabular}{|l|l|l|l|l|}
\hline No & Size $(\mathrm{cm})$ & location & Margin & Morphology \\
\hline 1 & Isointensity & Heterogeneous & Strong heterogeneous & Heterogeneous \\
\hline 2 & Isointensity & Heterogeneous & Moderate heterogeneous & Heterogeneous \\
\hline 3 & Hypointensity & Black-white & Strong heterogeneous & Heterogeneous \\
\hline 4 & Hypointensity & Heterogeneous & Strong homogeneous & Heterogeneous \\
\hline 5 & Isointensity & Heterogeneous & Moderate heterogeneous & Heterogeneous \\
\hline 6 & hypointensity & Heterogeneous & Strong heterogeneous & Heterogeneous \\
\hline 7 & hypointensity & Black-white & Strong heterogeneous & Heterogeneous \\
\hline
\end{tabular}

The detailed MRI findings of the seven cases are summarized in Tables 2 and 3. On MR imaging, six tumors showed mass effect. Four tumors showed a homogeneous isotensity signal intensity, and three tumors showed slightly low signal intensity on T1WI. All tumors illustrat- ed heterogeneous signal intensity on T2WI, two tumors showed substantial "black and white" patterns. Mixed hyperintense, isointense, and hypointense signal were seen in five lesions. Linear, curved and/or interweave low signal intensity lines within the lesions were found in all seven tumors.

Table 2. Magnetic resonance imaging findings from the seven intracranial solitary fibrous tumor cases.

\begin{tabular}{|l|l|l|l|l|}
\hline No & T1WI & T2WI & Enhancement pattern & DWI \\
\hline 1 & Isointensity & Heterogeneous & Strong heterogeneous & Heterogeneous \\
\hline 2 & Isointensity & Heterogeneous & Moderate heterogeneous & Heterogeneous \\
\hline 3 & Hypointensity & Black-white & Strong heterogeneous & Heterogeneous \\
\hline 4 & Hypointensity & Heterogeneous & Strong homogeneous & Heterogeneous \\
\hline 5 & Isointensity & Heterogeneous & Moderate heterogeneous & Heterogeneous \\
\hline 6 & hypointensity & Heterogeneous & Strong heterogeneous & Heterogeneous \\
\hline 7 & hypointensity & Black-white & Strong heterogeneous & Heterogeneous \\
\hline
\end{tabular}

Table 3. Magnetic resonance imaging findings from the seven intracranial solitary fibrous tumor cases.

\begin{tabular}{|l|l|l|l|l|l|l|l|l|l|}
\hline No & $\begin{array}{l}\text { Flow } \\
\text { void }\end{array}$ & $\begin{array}{l}\text { Low } \\
\text { signal } \\
\text { lines }\end{array}$ & $\begin{array}{l}\text { Mass } \\
\text { effect }\end{array}$ & $\begin{array}{l}\text { Cystic } \\
\text { change }\end{array}$ & Necrosis & edema & $\begin{array}{l}\text { Dural } \\
\text {-tail }\end{array}$ & $\begin{array}{l}\text { Ring-like } \\
\text { band }\end{array}$ & $\begin{array}{l}\text { Restricted } \\
\text { diffusion }\end{array}$ \\
\hline 1 & yes & yes & yes & periphery & No & No & Yes & yes & Yes \\
\hline 2 & yes & yes & yes & No & center & Yes & Yes & yes & No \\
\hline 3 & yes & yes & yes & No & No & Yes & No & yes & No \\
\hline 4 & yes & yes & no & no & No & No & No & no & No \\
\hline 5 & yes & yes & yes & periphery & center & Yes & No & yes & Yes \\
\hline 6 & yes & yes & yes & center & center & Yes & No & yes & Yes \\
\hline 7 & yes & yes & yes & no & no & yes & yes & yes & no \\
\hline
\end{tabular}


The lines llustrated an irregular interlace pattern, and were clearly showed on T2WI (Figure 1; Figure 2) Some lines might show unmarked on T1WI that needed to be careful observation and definition. Three tumors found cystic change areas in the peripheral region of the lesion, one tumor showed multiple cystic change areas within tumor.
All cystic change regions showed high signal on T2WI, low signal on T1WI, without enhancement on contrast enhance T1WI. Peri-tumor fluid collection was noted in three tumors. Seven tumors showed irregular flow voids within the tumor or at the periphery of the tumor clearly demonstrated on T2WI.

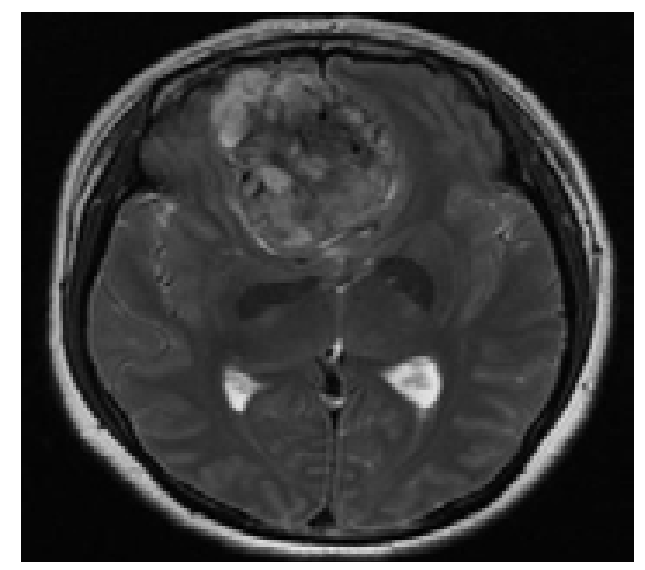

Figure 1. A 48-year-old woman with headache for 1 year: A.

T2WI axial MR image. Low signal intensity lines, and flow voids are clearly illustrated;

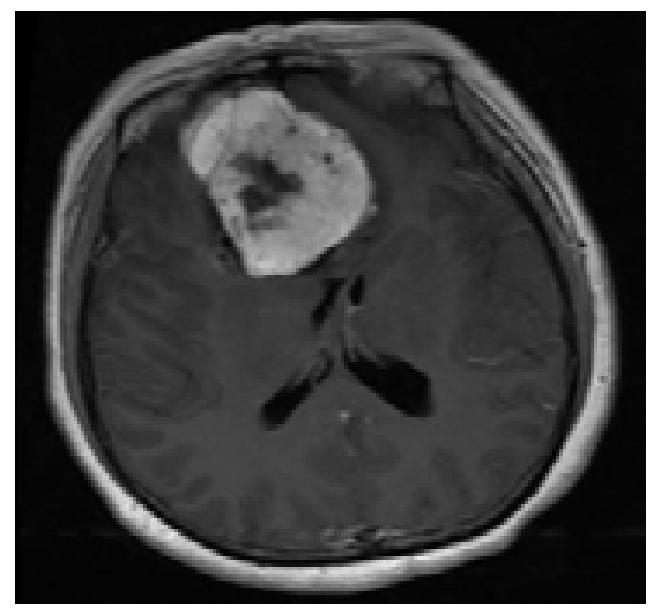

B. Contrast-enhanced image shows intense homogeneous enhancement; necrosis area is located in the central part of the tumor (without enhancement).

On gadopentetate dimeglumine enhanced imaging, all tumors showed intense enhancement, six tumors showed heterogeneous enhancement, and one showed homogeneous enhancement. Both low T2WI signal intensity areas and high T2WI signal intensity areas showed substantial enhancement. However, the degree of enhance- ment was related to the T2WI signal intensity. The higher T2WI signal intensity areas exhibited stronger enhancement compared to the low T2WI signal intensity areas. Necrosis areas within the central part of the tumor were seen on one tumor(Figure 1). Three tumors demonstrated a "dural tail" sign(Figure 2). Peri-tumoral edema was seen in four tumors. 


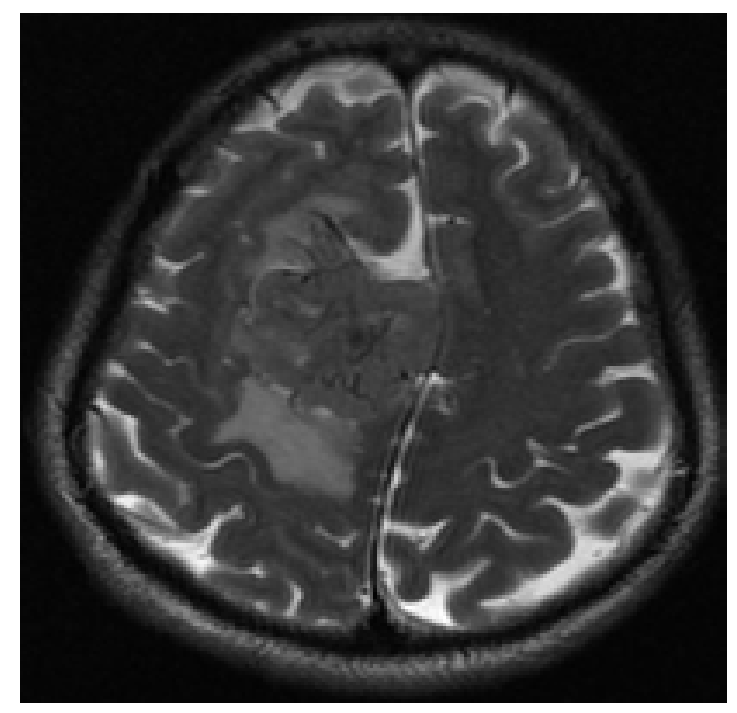

Figure 2. A 43-year-old man with headache and dizziness

for 1 month: A.T2WI axial MR image shows a large lobulated mass located near cerebral falx of the right side with homogenous isotensity signal intensity.

The irregular linear, strip low signal intensity lines are clearly illustrated.

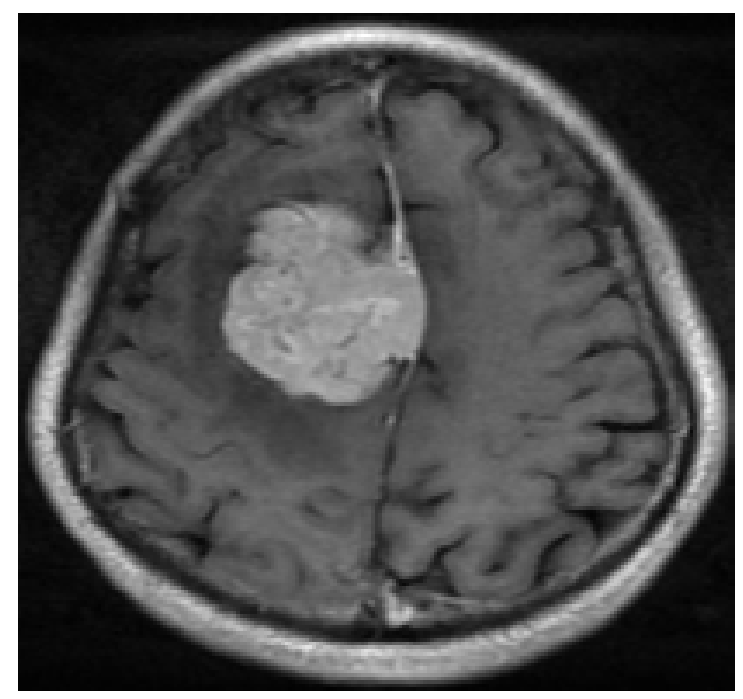

B. The tumor shows intensely homogeneous enhancement after Gadopentetate Dimeglumine administration.

Note: the "dural tail" sign(arrow)

On DWI, all tumors showed heterogeneous signal intensity. Marked high signal intensity restricted diffusion areas were found in three tumors. The high T2WI signal intensity areas showed slightly high signal intensity compared with low T2WI signal intensity areas on DWI. A ring-like high signal intensity band distributed around within the tumor was noted in six cases(Figure 2).

Microscopic examination, the tumors showed a fascicular proliferation pattern with irregular intercellular collagen bundles. The spindle and oval cell with ovoid nuclear and less cytoplasm were found in all tumors, they exhibited a patternless architecture. Immunohistochemical studies showed that $100 \%(7 / 7)$ tumors were CD 34 positive reaction, 100\% (7/7) were CD 99 positive reaction, 100\% (3/3) BcL-2 positive reaction, 57.1\% (4/7) vimenin positive reaction; S-100 negative reaction was noted in 100\% (4/4), and PR negative reaction in 100\% (4/4) were established. 


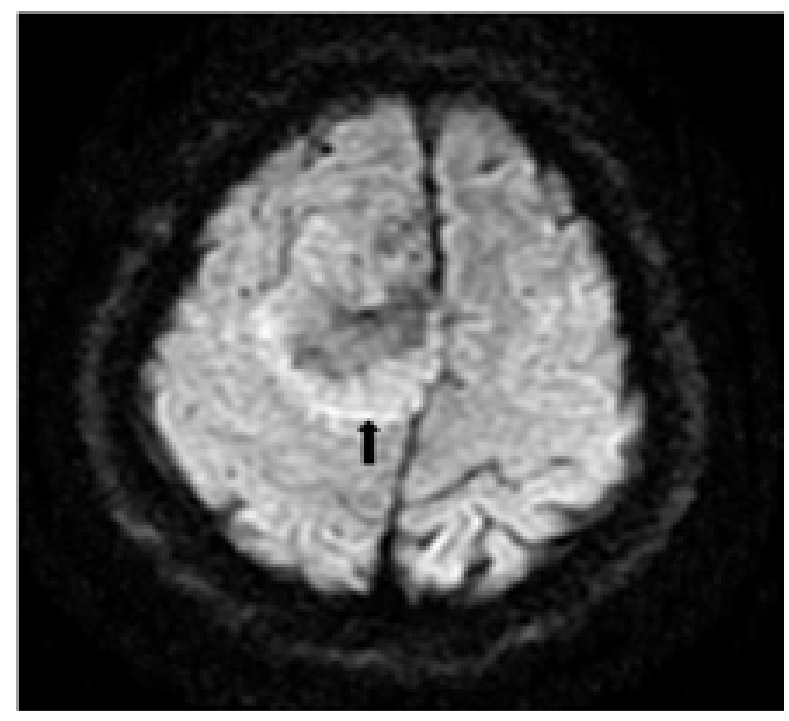

C. A ring-like high signal intensity band around the tumor is clearly demonstrated on DWI(arrow).

\section{Discussion}

Solitary fibrous tumor (SFT), a rare mesenchymal neoplasm, was first described by Klemperer and Rabin ${ }^{10}$. Primary SFT involving the central nervous system (CNS) was described as a distinct pathological entity in 1996 by Carneiro et $\mathrm{al}^{1}$, who reported five 5 ISFTs and two spinal SFTs that were distinguished from fibrous meningioma by pathological and immunohistochemical findings.

ISFT can occur at any age with the largest proportion occurring at 51-60 years. It occurs roughly equally in males and females ( $\mathrm{F}: \mathrm{M}=49.4 \%: 50.6 \%$ ). The mean greatest diameter is $4.9 \mathrm{~cm}$ (range $1.3-9 \mathrm{~cm})^{11}$. Majority of ISFTs $(87 \%)$ arise from the extra-axial space11, with a meningeal and a dura-based attachment ${ }^{1,7,12}$. However, intra-axial of brain parenchyma and ventricles have also been reported $^{3,7,13,14}$. The extra-axial tumors without connected with the dura were about of $13 \%{ }^{11}$. A review by Fargena et $\mathrm{al}^{12}$ showed that most ISFT were located along the tentorium cerebelli $(16 \%)$ followed by the frontal convexity, the cerebellopontine angle (CPA), the ventricles, the falx cerebri and the posterior fossa. It has been reported that $51 \%$ of ISFTs were supratentorial, and $34.4 \%$ were infratentorial11. Metellus et $\mathrm{al}^{15}$ reported that $33.3 \%$ solitary fibrous tumor of the central nervous system were located in the posterior fossa, supratentorial in $22.2 \%$. By contrast, in our small series, $85.7 \%(6 / 7)$ were supratentorial. The typical histologic features of ISFT are uniform spin- dle or oval cells arranged in a variable collagenous background or in interlacing fascicles, with prominent collagenous bands, and branching vascular channels with thin walls ${ }^{1,16}$. The amount of collagen and vascularity varies from tumor to tumor. Immunohistochemical study is a reliable method to identify the ISFT. The unique characteristic of SFTs is strong reactivity of the spindle cells for CD34 and a positive reaction for vimentin and BCL-2 EMA and S-100 are usually negative. The differential diagnosis of ISFT mainly includes fibroblastic meningioma and meningeal HPC. Although hemangiopericytoma also shows a positive reaction with vimentin and sometimes CD34, the reactivity is mild. Fibrous meningioma is usually positive for EMA (80\%) and negative (or mildly positive) for CD341, 3, 16. In our cases, 100\% (7/7) tumors were CD 34 positive reaction, 100\% (3/3) bcl-2 positive reaction; and 100\% (4/4) lesions were S-100 negative reaction.

On MR imaging, the ISFTs exhibited a diverse characteristics, with the appearance on the particular tissue components within the tumors. Generally, it was isointense on T1WI, and iso to hyperintense on T2WI, with intense heterogeneous or homogeneous enhancement ${ }^{17,18}$. Weon et al6 found that ISFTs mainly showed two different MR imaging features that was high or isosignal intensity on T1WI and low signal intensity on T2WI; and the isosignal 
or low signal intensity on T1WI and high signal intensity on T2WI.

Variable signal intensity showed on T2WI was noted by several studies, majority of tumors showed a heterogeneous pattern, a marked low signal intensity and high signal intensity presenting as a "black-and-white" mixed pattern has been accepted as an important feature in characterization of ISFT $^{6,16}$, these mixed signals were corresponded to the heterogeneous of areas with different cellularity, which was one of the pathological features of $\mathrm{SFT}^{19}$. In our group, two tumors illustrate a marked "black- and-white" pattern. Previous studies have also established that the varied MR images were mainly due to the different hypercellular and fibrous hypocellular component particular to the amount of collagen, fibroblasts and degeneration. The tumor signal intensity on the T2WIs decreased as the collagenous component increased. Thus, the T2WI was useful for excluding hemangiopericytomas, because it did not contain the areas of thick bands of collagen which appeared as hypointense on the T2WI ${ }^{17,18}$.

Interestingly, findings of our study showed that 100\% (7/7) of ISFTs had the linear, curved and/or interweave low signal intensity lines within the tumor. The lines were irregular interlace pattern clearly showed on T2WI. We presumed that these lines might be the bands of collagen or fibrosis ${ }^{17,18}$. Similar findings were also noted by previous investigators. Kim et $\mathrm{al}^{9}$ reported four 4 SFTs with that imaging feature. Pathological examination has established that those lines corresponded to the hypocellular collagenous stroma intervening in the hypercelluar area, and this hypocellular collagenous stroma was composed of a haphazard proliferation of spindle cells?. The authors recommended that hypointense lines were helpful in the differential diagnosis of masses involving the intracranial and extracranial head and neck regions. Based on a literature review and the results of our study, we believe that low signal intensity lines within the tumor may be a unique MR imaging feature of ISFTs, as ISFT contains collagen and fibrotic bands.

The contrast enhanced MR imaging also provided valuable imaging features in our study. All tumors demon- strated intense heterogeneous enhancement $(n=6)$ and homogeneous enhancement $(n=1)$. The enhancement degree of the lesions was associated with T2WI signal intensity. Although low T2WI signal intensity areas showed substantially intense enhancement, the enhancement degree of the higher T2WI signal intensity areas exhibited stronger than those of low T2WI signal intensity areas. Clarencon et al7 reported that the hypointense T2 signal intensity areas showed strong enhancement after contrast administration in the majority of ISFTs. But, this enhanced imaging feature was not showed in hemangiopericytomas ${ }^{6,7,9}$. The substantial intense enhancement was probably a result of rich vascularity of the SFT ${ }^{17,18}$. The "dural tail", a non-specific sign for any extra-axial lesion, is usually considered as one of main MRI characteristics in meningiomas. Absent this sign has been suggested to be a diagnostic clue for ISFT by some researcher ${ }^{7,15}$. Contrary, several studies demonstrated that the "dural tail"was not uncommon seen in ISFTs ${ }^{6,9,18}$. In our group, three 3tumors had this sign on contrast enhanced MRI.

DWI reflects the random thermal motion of molecules (Brownian motion). The ADC value is used to quantify the Brownian motion, and decreased movement of molecules in tissue correlates with a low ADC value. Recent studies have reported that ADC values and maps from DWI were very useful for detecting malignant lesions and evaluating their extent in SFT occurred in pleura ${ }^{20}$. In general, hyperintense areas on DWI present a restricted diffusivity. Based on pathological findings and literature review, Clarencon et $\mathrm{al}^{7}$ speculated that the hypercellular areas found in ISFTs might be responsible for the decreased ADC. In our study, three tumors had high signal intensity restricted diffusion areas. We also noted that all tumors demonstrated heterogeneous signal intensities on DWI, the intensity of the lesion on DWI also seemed to be related to the T2WI signal intensity. A ring-like high signal intensity band distributed around within the tumor on DWI was seen in five 5 cases. The restricted diffusion areas are hypercellular, and there are branching vascular channels with thin walls in the mesenchyme; these are considered focal hemangiopericytoma-like pattern areas $^{21}$. Therefore,even if DWI performance helps to obtain accurate diagnosis of ISFTs, nevertheless more information is required to confirm the diagnosis. 


\section{Conclusion}

ISFT is rare entity. Limited imaging information remains a diagnostic challenging. The accurate diagnosis is mainly relied on histopathological and immunohistochemical studies. Based on our current study, we can conclude that several MR image manifestations may provide effective evidence for diagnosis of ISFTs. The main diagnostic features on MR imaging are as follows: a well-defined, multilobular mass with homogeneous iso or hypointense signal on T1WI, variably heterogeneous on T2WI, T2 signal related intense enhancement, linear, curve, interweave low signal lines within the tumor, high signal restricted diffusion areas within tumor on DWI and a ring-like high signal intensity band around the tumor.

\section{Conflict of interest}

None.

\section{References}

1. Carneiro SS, Scheithauer BW, Nascimento AG, et al. Solitary fibrous tumor of the meninges: a lesion distinct from fibrous meningioma. A clinicopathologic and immunohistochemical study. Am J Clin Pathol. 1996;106:217224.

2. Yilmaz C, Kabatas S, Ozen OI, et al. Solitary fibrous tumor. J Clin Neurosci. 2009;16:1578-1581.

3. Tihan T, Viglione M, Rosenblum MK,et al. Solitary fibrous tumors in the central nervous system. A clinicopathologic review of 18 cases and comparison to meningeal hemangiopericytomas. Arch Pathol Lab Med. 2003;127:432-439.

4. Kasper E, Boruchow S, Lam FC, et al. "Hitting all the right markers to save a life" Solitary fibrous tumors of the central nervous system: Case series and review of the literature. Surg Neurol Int. 2012;3:83.

5. Boada M, Gomez E, Puig J,et al.Intraventricular fibrous tumor: a case report. Radiologia. 2009;51:512-515.

6. Weon YC, Kim EY, Kim HJ, et al. Intracranial solitary fibrous tumors: imaging findings in 6 consecutive patients. AJNR Am J Neuroradiol. 2007;28:1466-1469.

7. Clarencon F, Bonneville F, Rousseau A, et al. Intracranial solitary fibrous tumor: imaging findings. Eur J Radiol. 2011;80:387-394.

8. Bisceglia M, Dimitri L, Giannatempo G, et al. Solitary fibrous tumor of the central nervous system: report of an

African Health Sciences Vol 18 Issue 3, September, 2018 additional 5 cases with comprehensive literature review. Int J Surg Pathol. 2011;19:476-486.

9. Kim HJ, Lee HK, Seo JJ, et al. MR imaging of solitary fibrous tumors in the head and neck. Korean J Radiol. 2005;6:136-142.

10. Klemperer P RC. Primary neoplasms of the pleura.A report of five cases. Arch Pathol. 1992:385-412.

11. Bisceglia M, Galliani C, Giannatempo G, et al. Solitary fibrous tumor of the central nervous system: a 15-year literature survey of 220 cases (August 1996-July 2011). Adv Anat Pathol. 2011;18:356-392.

12. Fargen KM, Opalach KJ, Wakefield D, et al. The central nervous system solitary fibrous tumor: a review of clinical, imaging and pathologic findings among all reported cases from 1996 to 2010. Clin Neurol Neurosurg. 2011;113:703-710.

13. Kim KA, Gonzalez I, McComb JG, et al. Unusual presentations of cerebral solitary fibrous tumors: report of four cases. Neurosurgery. 2004; 54:1004-1009; discussion 9 .

14. Montano N, Doglietto F, Lauriola L, et al. Solitary fibrous tumour of the IV ventricle. Br J Neurosurg. 2010; 24:495-496.

15. Metellus P, Bouvier C, Guyotat J, et al. Solitary fibrous tumors of the central nervous system: clinicopathological and therapeutic considerations of 18 cases. Neurosurgery. 2007; 60:715-22; discussion 22.

16. Chan JK. Solitary fibrous tumour--everywhere, and a diagnosis in vogue. Histopathology. 1997;31:568-576.

17. Tateishi U, Nishihara H, Morikawa T, et al. Solitary fibrous tumor of the pleura: MR appearance and enhancement pattern. J Comput Assist Tomogr. 2002; 26:174-179.

18. Martin AJ, Fisher C, Igbaseimokumo U,et al. Solitary fibrous tumours of the meninges: case series and literature review. J Neurooncol. 2001;54:57-69.

19. Nawashiro H, Nagakawa S, Osada H, et al. Solitary fibrous tumor of the meninges in the posterior cranial fossa: magnetic resonance imaging and histological correlation--case report. Neurol Med Chir (Tokyo) .2000;40:432-434.

20. Inaoka T, Takahashi K, Miyokawa N, et al. Solitary fibrous tumor of the pleura: apparent diffusion coefficient (ADC) value and ADC map to predict malignant transformation. J Magn Reson Imaging. 2007;26:155-158.

21. Kim HJ, Kim HJ, Kim YD, et al.Solitary fibrous tumor of the orbit: CT and MR imaging findings. AJNR Am J Neuroradiol. 2008;29:857-862. 